\title{
Transmit Beamforming and Power Control for Cellular Wireless Systems
}

\author{
Farrokh Rashid-Farrokhi, Member, IEEE, K. J. Ray Liu, Senior Member, IEEE, and Leandros Tassiulas, Member, IEEE
}

\begin{abstract}
Joint power control and beamforming schemes are proposed for cellular systems where adaptive arrays are used only at base stations. In the uplink, mobile powers and receiver diversity combining vectors at base stations are calculated jointly. The mobile transmitted power is minimized, while the signal-tointerference-and-noise ratio (SINR) at each link is maintained above a threshold. A transmit diversity scheme for the downlink is also proposed where the transmit weight vectors and downlink power allocations are jointly calculated such that the SINR at each mobile is above a target value. The proposed algorithm achieves a feasible solution for the downlink if there is one and minimizes the total transmitted power in the network. In a reciprocal network it can be implemented in a decentralized system, and it does not require global channel response measurements. In a nonreciprocal network, where the uplink and downlink channel responses are different, the proposed transmit beamforming algorithm needs to be implemented in a centralized system, and it requires the knowledge of the downlink channel responses. The performances of these algorithms are compared with previously proposed algorithms through numerical studies.
\end{abstract}

Index Terms-Adaptive arrays, downlink beamforming, power control, transmit beamforming.

\section{INTRODUCTION}

$\mathbf{T}$ HE capacity of a cellular system is limited by the cochannel interference (CCI) and intersymbol interference (ISI). $\mathrm{CCI}$ is due to interference caused by users sharing the same channel. If the delay spread in a multipath channel is larger than a fraction of a symbol, the delayed components will cause ISI. Adaptive receiver beamforming schemes have been widely used to reduce both CCI and ISI and to improve the uplink capacity by adjusting the beam pattern such that the effective signal-to-interference-and-noise ratio (SINR) at the output of the beamformer is optimally increased. In order to reduce $\mathrm{CCI}$, the beamformer places nulls at the directions of interference, while the gain at the direction of the desired transmitter is maintained constant [1], [2]. In a single tap diversity, the signal from main path is considered as the signal of interest [1]. If multipath signals with large delay spread arrive at different angles, the single-tap beamformer rejects the ISI terms by placing nulls at the directions of multipath signals.

Manuscript received August 1997; revised May 1998. This paper was presented at CISS'97 and GLOBECOM'97. This work was supported in part by the NSF NYI Award MIP9457397, NSF Grant NCR-9406415, CAREER Award NCR-9502614.

F. Rashid-Farrokhi is with the Wireless Communications Research Department, Bell Labs, Lucent Technologies, Holmdel, NJ 07733, USA.

K. J. R. Liu and L. Tassiulas is with the Electrical Engineering Department and Institute for Systems Research, University of Maryland, College Park, MD 20742, USA.

Publisher Item Identifier S 0733-8716(98)07894-9.
By using space-time processing at the base stations, we can minimize CCI and ISI more effectively. Therefore, in mobile environments with large delay spread, the joint space-time processing will improve the performance [3].

Most often, deploying antenna arrays at mobiles is impractical. However, transmit diversity can be deployed at base stations to improve the downlink capacity. In scenarios where antenna arrays are used at transmitters, the beam-pattern of each antenna array can be adjusted to minimize the induced interference to undesired receivers. Transmit diversity and receiver beamforming are substantially different in nature. Receiver beamforming can be implemented independently at each receiver, without affecting the performance of other links, while transmit beamforming at each transmitter will change the interference to all other receivers. As a result, transmit beamforming has to be done jointly in the entire network. Moreover, in receiver beamforming, a local feedback from the receiver output is used to adjust the combining vector. In transmit beamforming, in order to measure the channel response, probing has to be done at the mobile [4], [5] and a feedback channel can be used to transmit the information to the base station. However, in time division duplex (TDD) systems where uplink and downlink channels are reciprocal, the uplink channel information can be used for the downlink [6]-[8].

In [4] and [6], transmit beamforming has been done by placing nulls at the direction of each cochannel receiver. This approach is well adapted to cases where the number of cochannels is less than the number of antenna elements. In [9], in a scenario where adaptive arrays are used at transmitters and receivers, an algorithm is proposed for selective transmission which uses the weight vectors calculated by single tap diversity combiners at the receivers. In [7] and [10], transmitter patterns are adjusted to minimize the overall interference to the other cochannel receivers using single tap transmit diversity. In [6], the use of multitap transmit diversity is proposed to reject the interference to other links. However, that work only considers the condition in which the number of available nulls is less than the number of antenna elements. The link quality is not guaranteed in any of the previous works. Also, by looking at adaptive beamforming between a transmitter and only its receiver, they ignored the possible effects that a change of beampattern in that transmitter could have on all receivers in the entire network.

In this paper we consider the problem of transmit beamforming within the context of the entire network. We introduce the notion of maximum achievable capacity in the downlink 
as the maximum number of users for which the SINR is achievable. Then, we propose an algorithm that jointly finds a set of feasible transmit beamforming weight vectors and downlink transmit power allocations such that the SINR at each link is greater than a target value. We will show that the proposed algorithm minimizes the total transmitted power in the network. We will extend the proposed algorithms to the case where multitap transmit diversity systems are used at the transmitters, which allows us to eliminate the ISI terms more effectively. However, multitap diversity systems suffer from larger peak-to-average-power ratio. The proposed algorithm can be used in time division multiple access (TDMA) or frequency division multiple access (FDMA) systems to reduce the reuse distance or increase the number of users in a cell. In code division multiple access (CDMA) systems, the presented schemes can be used to reduce the CCI and, as a result, to increase the capacity of a cell. The algorithms proposed in this paper can be easily extended to systems with macro-diversity, where antenna elements are located at different base stations. We will show that by adding optimal power allocations to the existing transmit beamforming algorithms, we can improve the performance significantly.

In a TDD system, where the transmit and receive channels are reciprocal, our algorithm is amenable to distributed implementation, and there is no need for global channel measurement in a network. In a frequency division duplex (FDD) system or a TDD system with large dwell time, the uplink and downlink channels are not reciprocal. In this case, our algorithm requires the downlink channel responses in order to calculate the downlink diversity vectors and power allocations. The downlink channel characteristic is measured at the mobile and transmitted to the base station through a feedback channel. Using our method, a centralized processor is able to calculate feasible combining weight vectors using the global channel measurements.

The organization of paper is as follows: in Section II we will present our model of a network with multipath fading channels and discuss receiver beamforming algorithms. In Section III, the joint power control and diversity combining for uplink is presented. In Section IV we will describe our transmit diversity algorithm and prove its optimality and convergence. We will also propose suboptimal algorithms that require smaller amounts of global channel measurements. In Section $\mathrm{V}$ we will evaluate the performance of our algorithms using simulation study. In the Appendix, first the algorithm proposed in [11] is extended to the multitap receiver diversity combiner. We will then derive the formulation for the multitap transmit beamforming and will show that the same algorithms can be applied to this case.

\section{SyStem Model AND ReCEIVE DIVERSITY COMBINING}

Consider a set of cochannel links which may share base stations as in CDMA systems or may use distinct base stations as in TDMA networks. Each link consists of a mobile and its assigned base station. Assume that for each link there are maximum $L$ paths, and that coherent detection is possible so that it is sufficient to model this multiuser system by an equivalent base band model. We assume antenna arrays with $P$ elements are used only at base stations. With that as given and the slow fading assumption, the received signal at the $i$ th base station, denoted by $\boldsymbol{x}_{i}(t)$, is given by [13]

$$
\begin{aligned}
\boldsymbol{x}_{i}(t)= & \sum_{m=1}^{M} \sum_{l=1}^{L} \sqrt{\rho_{m i} G_{m i} \alpha_{m i}^{l} P_{m}} \boldsymbol{a}_{m i}\left(\theta_{l}\right) \\
& \cdot u_{m i}\left(t-\tau_{m i}^{l}\right)+\boldsymbol{n}_{i}(t)
\end{aligned}
$$

where $\rho_{m i}$ models the log-normal shadow fading, $\alpha_{m i}^{l}$ and $G_{m i}$ are the $l$ th path fading and loss from the $m$ th mobile to the $i$ th base station, respectively. $P_{m}$ is the transmitted power by the $m$ th mobile. $\boldsymbol{a}_{m i}\left(\theta_{l}\right)=\left[a_{m i}^{1}\left(\theta_{l}\right) \cdots a_{m i}^{P}\left(\theta_{l}\right)\right]^{T}$ is the $i$ th array response to the signal coming from the $m$ th mobile at direction $\theta_{l} \cdot \boldsymbol{n}_{i}(t)$ is the thermal noise vector at the $i$ th base station array. The signal $u_{m i}(t)$ can be expressed as a function of the message symbols $s_{m}(n)$ by

$$
u_{m i}(t)=\sum_{n=0}^{k} g_{m}(t-n T) s_{m}(n)
$$

where $k=\lfloor t / T\rfloor$, and $g_{m}(t)$ models the effect of the pulseshaping function of the modulation scheme. $T$ is the symbol duration. We rewrite the signal at the output of the array as

$$
\begin{aligned}
\boldsymbol{x}_{i}(t)= & \sum_{m=1}^{M} \sum_{n=0}^{k} \sum_{l=1}^{L} \sqrt{\rho_{m i} G_{m i} \alpha_{m i}^{l} P_{m}} \boldsymbol{a}_{m i}\left(\theta_{l}\right) \\
& \cdot g_{m}\left(t-n T-\tau_{m i}^{l}\right) s_{m}(n)+\boldsymbol{n}_{i}(t)
\end{aligned}
$$

Now define the impulse response from the $m$ th mobile to the $p$ th element of the $i$ th base station by

$$
h_{m i}^{p}(n)=\sum_{l=1}^{L} \sqrt{\rho_{m i} G_{m i} \alpha_{m i}^{l}} a_{m i}^{p}\left(\theta_{l}\right) r_{m i}^{p l}(n)
$$

where $r_{m i}^{p l}(n)$ includes the effect of the transmitter and receiver filter and $g_{m}(t)$. The vector channel impulse response for the equivalent discrete model is given by

$$
h_{m i}(n)=\left[h_{m i}^{1}(n), \cdots, h_{m i}^{P}(n)\right]^{T}
$$

Assume that the length of the impulse response is $N$. Then we can express the sampled received signal vector as

$$
\boldsymbol{x}_{i}(k)=\sum_{m=1}^{M} \sum_{n=0}^{N-1} \boldsymbol{h}_{m i}(n) \sqrt{P_{m}} s_{m}(k-n)+\boldsymbol{n}_{i}(k)
$$

where $\boldsymbol{n}_{i}(k)=\left[n_{i}^{1}(k), n_{i}^{2}(k), \cdots, n_{i}^{p}(k)\right]^{T}$, and $n_{i}^{p}(k)$ is the sampled thermal noise at the output of the matched filter at the $k$ th element of the array. We are more interested in the matrix presentation of the channel, and multiplication instead of convolution. Therefore we define the channel response matrix $\boldsymbol{H}_{m i}$ as

$$
\boldsymbol{H}_{m i}=\left[\boldsymbol{h}_{m i}(0), \cdots, h_{m i}(N-1)\right]
$$




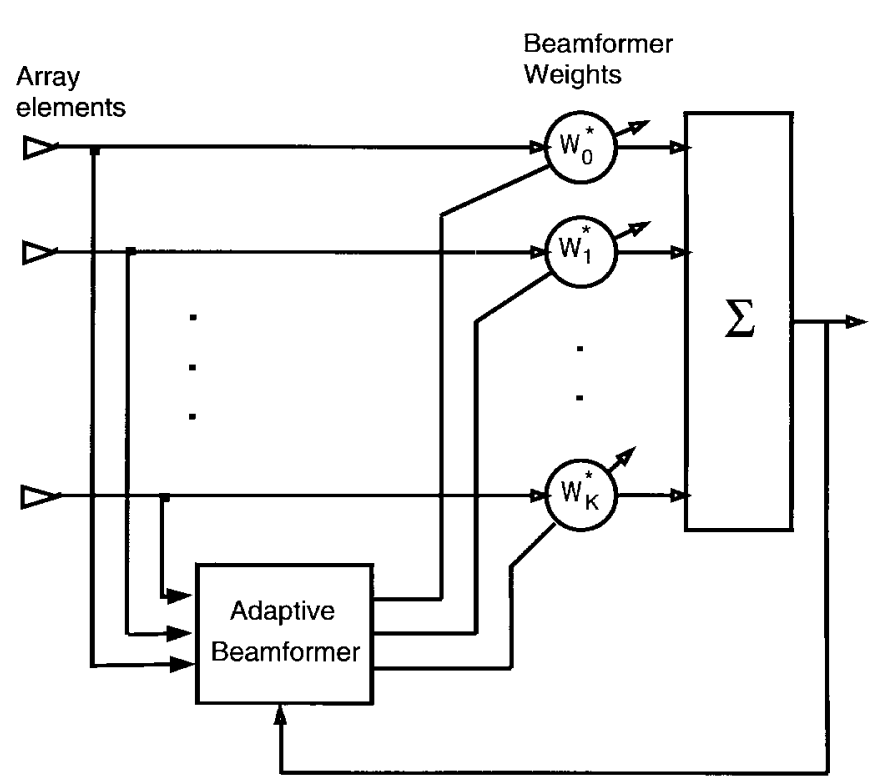

Fig. 1. Block diagram of a receive diversity system.

and modify (1) as

$$
\boldsymbol{x}_{i}(k)=\sum_{m=1}^{M} \boldsymbol{H}_{m i} \sqrt{P_{m}} \boldsymbol{s}_{m}(k)+\boldsymbol{n}_{i}(k)
$$

where $s_{i}(k)=\left[s_{i}(k), \cdots, s_{i}(k-N+1)\right]^{T}$.

The block diagram of a system with adaptive arrays at receivers is shown in Fig. 1. The output of the combiner at the $i$ th receiver is written as $\boldsymbol{e}_{i}(k)=\boldsymbol{w}_{i}^{H} \boldsymbol{x}_{i}(k)$, where $\boldsymbol{w}_{i}$ is the beamforming weight vector. The average output power is given by

$$
\begin{aligned}
\mathcal{E}_{i} & =\mathrm{E}\left\{\boldsymbol{w}_{i}^{H} \boldsymbol{x}_{i}(k) \boldsymbol{x}_{i}^{H}(k) \boldsymbol{w}_{i}\right\} \\
& =\boldsymbol{w}_{i}^{H} \mathrm{E}\left\{\boldsymbol{x}_{i}(k) \boldsymbol{x}_{i}^{H}(k)\right\} \boldsymbol{w}_{i}=\boldsymbol{w}_{i}^{H} \boldsymbol{\Phi}_{i} \boldsymbol{w}_{i} .
\end{aligned}
$$

The aim in receiver beamforming is to adjust the weight vectors to achieve maximum SINR at the output of the combiner. This can be achieved by minimizing the total interference at the output of beamformer while the gain for the desired user is kept constant. The optimum weight vector is then calculated by the following minimization:

$$
\begin{aligned}
& \min _{\boldsymbol{w}_{i}} \boldsymbol{w}_{i}^{H} \boldsymbol{\Phi}_{i} \boldsymbol{w}_{i}, \\
& \text { subject to } \boldsymbol{w}_{i}^{H} \boldsymbol{h}_{i i}(0)=1 .
\end{aligned}
$$

The solution to this problem, known as minimum variance distortionless response (MVDR), is given by [1]

$$
\hat{\boldsymbol{w}}_{i}=\frac{\Phi_{i}^{-1} \boldsymbol{h}_{i i}(0)}{\boldsymbol{h}_{i i}^{H}(0) \Phi_{i}^{-1} \boldsymbol{h}_{i i}(0)} .
$$

Here the channel response to the desired user is assumed to be known. If the channel response is not available, we use a training sequence, which is correlated with the desired signal. The weight vector is obtained by minimizing the mean square error between the beamformer output and the training sequence, denoted by $d_{i}$ [1]. The minimization problem is defined as

$$
\hat{\boldsymbol{w}}_{i}=\arg \min _{\boldsymbol{w}_{i}} E\left\{\left|d_{i}-\boldsymbol{w}_{i}^{H} \boldsymbol{x}_{i}\right|^{2}\right\} .
$$

The solution to the above minimum mean square error (MMSE) problem is given by the Wiener-Hopf solution

$$
\hat{\boldsymbol{w}}_{i}=\Phi_{i}^{-1} \boldsymbol{p}_{i}
$$

where

$$
\boldsymbol{p}_{i}=E\left\{\boldsymbol{x}_{i} d_{i}^{*}\right\} .
$$

For simplicity, the training sequence is considered to be a copy of the signal of interest. Then it can be shown [1] that

$$
\boldsymbol{w}_{i}=\sqrt{P_{i}} \Phi_{i}^{-1} h_{i i}(0) .
$$

Both MVDR (7) and MMSE (8) solutions can be expressed as $\boldsymbol{w}_{i}=c \Phi_{i}^{-1} \boldsymbol{h}_{i i}(0)$, where $c$ is a constant and does not affect the SINR at the output of beamformer. It can be shown that both methods maximize the SINR [1]. The weight vectors are adjusted during the transmission of the training sequence and are kept constant in between training phases. The frequency of updating the weight vectors should be high enough such that the channel response can be considered constant between the training phases. There are computationally efficient and adaptive techniques to update weight vectors such as least mean square (LMS) [18] or recursive least square (RLS) [19].

\section{JOINT POWER CONTROL AND RECEIVE DIVERSITY COMBINING}

In a network with power control capability, the transmitted power is updated based on the link quality at its receiver, e.g., SINR or bit error rate (BER). In this work we consider the SINR-based power control schemes [16]. The SINR is a function of receive diversity combining vector at each receiver. On the other hand, the diversity combining weight vectors also depend on the transmitted powers. This fact implies that in order to achieve the optimal performance, we need to consider the joint calculation of combining vectors and allocated powers in a network [12].

First we evaluate the SINR at each combiner output as a function of the gain matrix $H_{m i}$, weight vector $w_{i}$, and transmitted powers. Assuming that the transmitted signals from different sources are uncorrelated and zero mean, and the additive noise is spatially and temporally white, we can write the correlation matrix as

$$
\begin{aligned}
\Phi_{i} & =E\left\{\boldsymbol{x}_{i} \boldsymbol{x}_{i}^{H}\right\} \\
& =\sum_{m=1}^{M} P_{m} E\left\{\boldsymbol{H}_{m} \boldsymbol{s}_{m}(k) \boldsymbol{s}_{m}^{H}(k) \boldsymbol{H}_{m}^{H}\right\}+E\left\{\boldsymbol{n}_{i}(k) \boldsymbol{n}_{i}^{H}(k)\right\} \\
& =\sum_{m=1}^{M} P_{m} \boldsymbol{H}_{m i} \boldsymbol{H}_{m i}^{H}+N_{i} \boldsymbol{I} \\
& =\sum_{m=1}^{M} P_{m} \sum_{n=0}^{N-1} h_{m i}(n) h_{m i}^{H}(n)+N_{i} \boldsymbol{I}=\Phi_{i}^{s}+\Phi_{i}^{I}
\end{aligned}
$$


where $\Phi_{i}^{s}$ and $\Phi_{i}^{I}$ are the correlation matrix of the desired signal and the interference, respectively, defined as

$$
\Phi_{i}^{s}=P_{i} h_{i i}(0) h_{i i}^{H}(0)
$$

and

$$
\Phi_{i}^{I}=\sum_{n \neq 0} P_{i} \boldsymbol{h}_{i i}(n) \boldsymbol{h}_{i i}^{H}(n)+\sum_{j \neq i} P_{j} \boldsymbol{H}_{m i} \boldsymbol{H}_{m i}^{H}+N_{i} \boldsymbol{I} .
$$

Define the gain matrix $\boldsymbol{G}_{m i}=\boldsymbol{H}_{m i} \boldsymbol{H}_{m i}^{H}$. The gain matrix $\boldsymbol{G}_{i i}$ can be separated into signal and interferences matrices

$$
\boldsymbol{G}_{i i}=G_{i i}^{s}+G_{i i}^{I}
$$

where $G_{i i}^{s}=\boldsymbol{g}_{i i} \boldsymbol{g}_{i i}^{H}$ and $\boldsymbol{g}_{i i}=\boldsymbol{h}_{i i}(0)$. The desired signal power at the output of the beamformer is given by $\boldsymbol{w}_{i}{ }^{H} G_{i i}^{s} \boldsymbol{w}_{i}$, and the interference power from the $m$ th mobile is $\boldsymbol{w}_{i}{ }^{H} \boldsymbol{G}_{m i}^{T} \boldsymbol{w}_{i}$ where

$$
\boldsymbol{G}_{m i}^{I}= \begin{cases}\boldsymbol{G}_{m i}, & m \neq i \\ \boldsymbol{G}_{i i}-\boldsymbol{G}_{i i}^{s}, & \text { otherwise. }\end{cases}
$$

The SINR at the beamformer output can be written as

$$
\Gamma_{i}=\frac{\boldsymbol{w}_{i}^{H} \Phi_{i}^{s} \boldsymbol{w}_{i}}{\boldsymbol{w}_{i}^{H} \Phi_{i}^{I} \boldsymbol{w}_{i}}=\frac{P_{i} \boldsymbol{w}_{i}^{H} \boldsymbol{G}_{i i}^{s} \boldsymbol{w}_{i}}{\sum_{m} P_{m} \boldsymbol{w}_{i}^{H} G_{m i}^{I} \boldsymbol{w}_{i}+N_{i} \boldsymbol{w}_{i}^{H} \boldsymbol{w}_{i}} .
$$

In order to provide the required link quality, the SINR at link $i$ should be at least $\gamma_{i}$. Consider a beamforming vector set $\boldsymbol{A}=\left\{\boldsymbol{w}_{1}, \cdots, \boldsymbol{w}_{M}\right\}$. A set of cochannel links is feasible if there exists a power vector $\boldsymbol{P}=\left[P_{1}, \cdots, P_{M}\right]^{T}$ and a set $\boldsymbol{A}$ such that the link quality is satisfied for each link. That is,

$$
\Gamma_{i} \geq \gamma_{i}
$$

For a fixed diversity combiner, the optimum transmitted power is achieved when $\Gamma_{i}=\gamma_{i}$. The minimum power allocation can be achieved by iterative power control schemes which are proposed for systems with fixed gain antennas [11]

$$
\begin{array}{r}
P_{i}^{n+1}=\gamma_{i} P_{i}^{n} \frac{\boldsymbol{w}_{i}^{H} \Phi_{i}^{I} \boldsymbol{w}_{i}}{\boldsymbol{w}_{i}^{H} \Phi_{i}^{s} \boldsymbol{w}_{i}} \\
=\gamma_{i} \frac{\sum_{m} P_{m}^{n} \boldsymbol{w}_{i}^{H} \boldsymbol{G}_{m i}^{I} \boldsymbol{w}_{i}+N_{i} \boldsymbol{w}_{i}^{H} \boldsymbol{w}_{i}}{\boldsymbol{w}_{i}^{H} G_{i i}^{S} \boldsymbol{w}_{i}}, \\
(i=1, \cdots, M)
\end{array}
$$

where $P_{i}^{n}$ is the transmitted power at the $n$th iteration by the $i$ th mobile. Similar to systems with fixed gain antennas, we can show that the above iteration starting from an arbitrary power vector $\boldsymbol{P}^{0}$ converges to the optimum power allocation for the desired SINR. Now we define the problem as to find a set of beamforming vectors and power allocations to minimize the total transmitted power while the link quality is maintained at each link

$$
\min _{A, P} \sum_{i} P_{i}
$$

such that $\Gamma_{i} \geq \gamma_{i}$.

In [11], we have shown that if the set of cochannel links is feasible, there exists a unique set of weight vectors and power allocations such that the transmitted powers are minimal among all feasible solutions. In [12], the following iterative algorithm has been proposed to find the joint power allocation and combining vectors. The algorithm steps at the $n$th iteration are given as follows.

\section{Algorithm A:}

1) The beamforming step, which is equivalent to maximizing the SINR

$$
\begin{aligned}
\boldsymbol{w}_{i}^{n} & =\arg \max _{\boldsymbol{w}_{i}} \frac{\boldsymbol{w}_{i}^{H} \Phi_{i}^{s} \boldsymbol{w}_{i}}{\boldsymbol{w}_{i}^{H} \Phi_{i}^{I} \boldsymbol{w}_{i}} \\
& =\arg \max _{\boldsymbol{w}_{i}} \frac{P_{i} \boldsymbol{w}_{i}^{H} \boldsymbol{G}_{i i}^{s} \boldsymbol{w}_{i}}{\sum_{m} P_{m}^{n} \boldsymbol{w}_{i}^{H} \boldsymbol{G}_{m i}^{I} \boldsymbol{w}_{i}+N_{i} \boldsymbol{w}_{i}^{H} \boldsymbol{w}_{i}} .
\end{aligned}
$$

2) Transmitted power is updated by

$$
\begin{aligned}
P_{i}^{n+1} & =\gamma_{i} P_{i}^{n} \frac{\left(\boldsymbol{w}_{i}^{n}\right)^{H} \Phi_{i}^{I} \boldsymbol{w}_{i}^{n}}{\left(\boldsymbol{w}_{i}^{n}\right)^{H} \Phi_{i}^{s} \boldsymbol{w}_{i}^{n}} \\
& =\gamma_{i} \frac{\sum_{m} P_{m}^{n}\left(\boldsymbol{w}_{i}^{n}\right)^{H} \boldsymbol{G}_{m i}^{I} \boldsymbol{w}_{i}^{n}+N_{i}\left(\boldsymbol{w}_{i}^{n}\right)^{H} \boldsymbol{w}_{i}^{n}}{\left(\boldsymbol{w}_{i}^{n}\right)^{H} \boldsymbol{G}_{i i}^{s} \boldsymbol{w}_{i}^{n}} .
\end{aligned}
$$

In [11], we have shown that starting from an arbitrary power vector $\boldsymbol{P}^{0}$, the above algorithm converges to the optimal power allocations and combining vectors such that the transmitted power is minimized among all feasible power allocations and combining vectors. A detailed proof of the convergence of the algorithm can be found in [11]. Algorithm A can be applied to systems where multitap diversity combiners are used at receivers. The formulation and detailed discussion of this case can be found in the Appendix.

\section{TRANSMIT BEAMFORMING}

In the following we assume that only base stations use antenna arrays. We will present an algorithm that finds a set of transmit weight vectors and power allocations such that the desired SINR at each mobile is above a target value. As we will see later, the transmitted power is also controlled by the transmit diversity weight vectors. We will show that the proposed algorithm minimizes the total transmitted power in the network as well.

The block diagram of a transmit beamforming system is shown in Fig. 2. Note that a base station may transmit to more than one mobile with different beamforming weight vectors. Denote the diversity vector for the $i$ th mobile by $\boldsymbol{w}_{i}$. The received signal at each mobile is a superposition of the transmitted signal from different base stations and their delayed versions through the multipath channel. The received signal at the $i$ th mobile is then given by

$$
\begin{aligned}
\tilde{y}_{i}(t)= & \sum_{b=1}^{M} \sum_{n=0}^{k} \sum_{l=1}^{L} \boldsymbol{w}_{b}^{H} \boldsymbol{a}_{i b}\left(\theta_{l}\right) \\
& \cdot \sqrt{\rho_{i b} G_{i b} \alpha_{i b}^{l}} g_{i b}\left(t-n T-\tau_{i b}^{l}\right) \sqrt{\tilde{P}_{b}} \tilde{s}_{b}(n)+\tilde{n}_{i}(t)
\end{aligned}
$$

where $k=\lfloor t / T\rfloor, \tilde{s}_{b}$ is the message signal transmitted from the $b$ th base station to its associated mobile, and $\tilde{n}_{i}(t)$ is the thermal noise at the $i$ th mobile. $\tilde{P}_{b}$ can be considered as the 


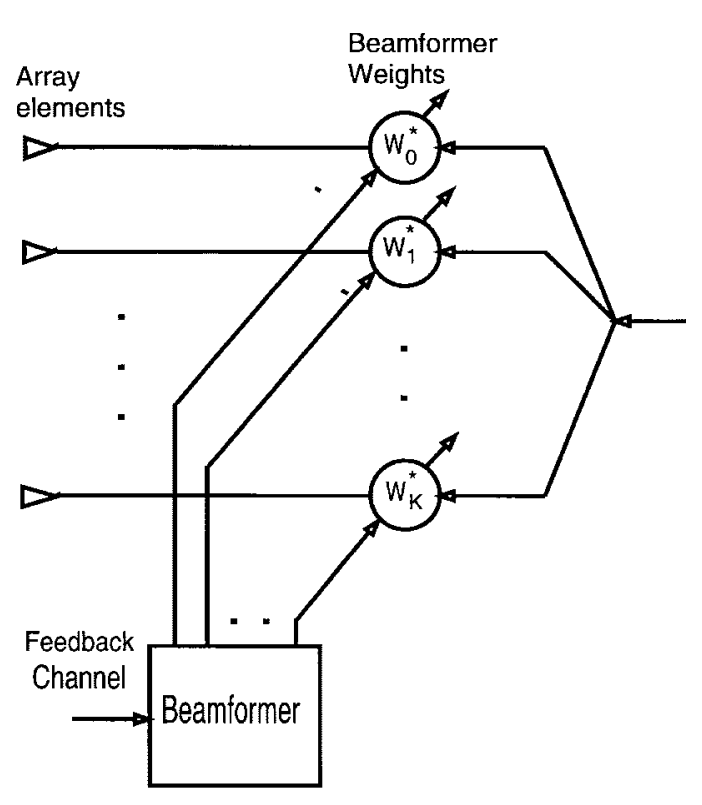

Fig. 2. Block diagram of a transmit diversity system.

signal power before the beamformer. Instead of absorbing this factor into the beamforming weight vector, we use it to adjust the level of the transmit power. The channel can be modeled by a discrete impulse response given by

$$
h_{i b}(n)=\sum_{l=1}^{L} a_{i b}\left(\theta_{l}\right) \sqrt{\rho_{i b} G_{i b} \alpha_{i b}^{l}} r_{i b}^{l}(n)
$$

where $r_{i b}^{l}(n)$ includes the effect of the receiver matched filter, wave shaping function, and transmitter filter. Assuming that the length of the discrete equivalent impulse response is $N$, we can express the sampled version of (11) at the symbol interval as

$$
\tilde{z}_{i}(k)=\sum_{b=1}^{M} \sum_{n=0}^{N-1} \boldsymbol{w}_{b}^{H} \boldsymbol{h}_{i b}(n) \sqrt{\tilde{P}_{b}} \tilde{s}_{b}(k-n)+\tilde{n}_{i}(k) .
$$

It follows that

$$
\tilde{z}_{i}(k)=\sum_{b=1}^{M} \boldsymbol{w}_{b}^{H} \boldsymbol{H}_{i b} \sqrt{\tilde{P}_{b}} \tilde{\boldsymbol{s}}_{b}(k)+\tilde{n}_{i}(k)
$$

where $\tilde{\boldsymbol{s}}_{b}(k)=\left[\tilde{\boldsymbol{s}}_{b}(k), \cdots, \tilde{s}_{b}(k-N+1)\right]^{T}$. Define $\tilde{\boldsymbol{x}}_{i b}(k)=$ $\boldsymbol{H}_{i b} \sqrt{\tilde{P}_{b}} \tilde{\boldsymbol{s}}(k)$. Then the received signal at the $i$ th receiver is expressed as

$$
\tilde{z}_{i}(k)=\sum_{b} \boldsymbol{w}_{b}^{H} \tilde{\boldsymbol{x}}_{i b}(k)+\tilde{n}_{i}(k)
$$

Similar to the receive diversity case, we can show that the desired signal power at the $i$ th receiver is given by $\tilde{P}_{i} \boldsymbol{w}_{i}^{H} G_{i i}^{s} \boldsymbol{w}_{i}$, and the interference power from the $b$ th base is given by $\tilde{P}_{b} \boldsymbol{w}_{b}^{H} \boldsymbol{G}_{i b}^{I} \boldsymbol{w}_{b}$, where $\boldsymbol{G}_{i i}^{s}$ and $\boldsymbol{G}_{i b}^{I}$ are defined as in the uplink. The SINR at this receiver is given by

$$
\Gamma_{i}=\frac{\tilde{P}_{i} \boldsymbol{w}_{i}^{H} \boldsymbol{G}_{i i}^{s} \boldsymbol{w}_{i}}{\sum_{b} \tilde{P}_{b} \boldsymbol{w}_{b}^{H} \boldsymbol{G}_{i b}^{I} \boldsymbol{w}_{b}+\tilde{N}_{i}}
$$

where $\tilde{N}_{i}$ is the thermal noise power at the $i$ th mobile.
In contrast to the uplink case, there is no solution that minimizes the transmitted power by each base station. Later, we will show this fact by a simple counter example. The transmit power by the $i$ th base station is given by $\tilde{P}_{i}\left\|\boldsymbol{w}_{i}\right\|^{2}$. We define the problem as a total network power minimization problem

$$
\begin{aligned}
& \min _{\tilde{\boldsymbol{P}}, \boldsymbol{A}} \sum_{i} \tilde{P}_{i}\left\|w_{i}\right\|^{2}, \\
& \text { subject to } \Gamma_{i} \geq \gamma_{i} .
\end{aligned}
$$

The solution to this problem can be found by standard optimization methods. However, in the following we will present an algorithm that achieves the optimal solution. The minimum power is achieved when the SINR is equal to the target value. That is,

$$
\Gamma_{i}=\gamma_{i}, \quad(i=1, \cdots, M)
$$

which in matrix form can be written as

$$
\tilde{\boldsymbol{P}}=\boldsymbol{D}_{w} \boldsymbol{F}_{w}^{T} \tilde{\boldsymbol{P}}+\tilde{\boldsymbol{u}}_{w}
$$

where $\boldsymbol{D}_{w}$ and $\boldsymbol{F}_{w}$ are defined as

$$
\begin{aligned}
{\left[\boldsymbol{F}_{w}\right]_{i j} } & =\boldsymbol{w}_{i}^{H} \boldsymbol{G}_{j i}^{I} \boldsymbol{w}_{i} \\
\boldsymbol{D}_{w} & =\operatorname{diag}\left\{\frac{\gamma_{1}}{\boldsymbol{w}_{1}^{H} \boldsymbol{G}_{11}^{s} \boldsymbol{w}_{1}}, \cdots, \frac{\gamma_{M}}{\boldsymbol{w}_{M}^{H} \boldsymbol{G}_{M M}^{s} \boldsymbol{w}_{M}}\right\}
\end{aligned}
$$

and

$$
\left[\tilde{\boldsymbol{u}}_{w}\right]_{i}=\frac{\gamma_{i} \tilde{N}_{i}}{\boldsymbol{w}_{i}^{H} \boldsymbol{G}_{i i}^{s} \boldsymbol{w}_{i}}
$$

In the following we consider the problem of the joint computation of a feasible set of combining weight vectors and power allocations. We will propose an algorithm that achieves the optimal solution if there exists at least one feasible solution. First we construct a virtual uplink network whose channel responses are similar to that of the downlink. Then we find the receiver diversity vectors at the base stations of the virtual uplink, and at each iteration we use the same combining vector for the downlink. The algorithm steps at the $n$th iteration are as follows.

\section{Algorithm B:}

1) Diversity combining for virtual uplink:

$$
\begin{aligned}
\boldsymbol{w}_{i}^{n} & =\arg \max _{\boldsymbol{w}_{i}} \frac{\boldsymbol{w}_{i}^{H} \Phi_{i}^{s} \boldsymbol{w}_{i}}{\boldsymbol{w}_{i}^{H} \Phi_{i}^{I} \boldsymbol{w}_{i}} \\
& =\arg \max _{\boldsymbol{w}_{i}} \frac{P_{i}^{n} \boldsymbol{w}_{i}^{H} G_{i i}^{s} \boldsymbol{w}_{i}}{\sum_{m} P_{m}^{n} \boldsymbol{w}_{i}^{H} \boldsymbol{G}_{m i}^{I} \boldsymbol{w}_{i}+\boldsymbol{w}_{i}^{H} \boldsymbol{w}_{i}}
\end{aligned}
$$

2) Virtual uplink power update $\left(\boldsymbol{P}^{n+1}\right)$ :

$$
\boldsymbol{P}^{n+1}=\boldsymbol{D}_{w}[n] \boldsymbol{F}_{w}[n] \boldsymbol{P}^{n}+\boldsymbol{u}_{w}[n]
$$

3) Downlink power update $\left(\tilde{\boldsymbol{P}}^{n+1}\right)$ :

$$
\tilde{\boldsymbol{P}}^{n+1}=\boldsymbol{D}_{w}[n] \boldsymbol{F}_{w}^{T}[n] \tilde{\boldsymbol{P}}^{n}+\tilde{\boldsymbol{u}}_{w}[n] .
$$


In the above algorithm, $\boldsymbol{w}_{i}^{n}$ is the optimal beamforming for the power allocation at the $n$th iteration. $\boldsymbol{F}_{w}[n], \boldsymbol{D}_{w}[n]$, and $\tilde{\boldsymbol{u}}_{w}[n]$ are defined as in (15) where $\boldsymbol{w}_{i}$ is replaced by $\boldsymbol{w}_{i}^{n}$, and $\boldsymbol{u}_{w}[n]$ is defined as

$$
\left[\boldsymbol{u}_{w}[n]\right]_{i}=\frac{\gamma_{i}\left\|\boldsymbol{w}_{i}^{n}\right\|^{2}}{\left(\boldsymbol{w}_{i}^{n}\right)^{H} \boldsymbol{G}_{i i}^{s} \boldsymbol{w}_{i}^{n}}
$$

The extension of this algorithm for multitap transmit diversity systems is presented in the Appendix.

\section{A. Convergence of the Algorithm}

In order to show that the above algorithm converges to a feasible solution for the downlink, we first use the fact that the uplink power allocation and beamforming vectors converge to a constant (optimal) power allocation. Then we conclude that the uplink gain matrix converges to a fixed matrix whose eigenvalues are inside the unit circle. Using the fact that the eigenvalues of the gain matrix in the downlink and uplink are the same, we conclude that the downlink iteration is convergent if the virtual uplink is so. First we introduce the definition of an asymptotically constant system. An asymptotically constant system is defined as follows.

Definition: The following linear system:

$$
\boldsymbol{x}[n+1]=\boldsymbol{C}[n] \boldsymbol{x}[n]+\boldsymbol{d}[n]
$$

is called asymptotically constant if

$$
\boldsymbol{C}[n]=\boldsymbol{C}+\boldsymbol{E}[n]
$$

and

$$
\lim _{n \rightarrow \infty}\|\boldsymbol{E}[n]\|=0 .
$$

The stability of these systems can be evaluated using the following theorem [15].

Theorem 1: The asymptotically constant system is asymptotically stable if the matrix $C$ has all its eigenvalues inside the unit circle.

In the following we will show that the downlink power control and transmit beamforming system proposed in Algorithm B is an asymptotically stable system. The first two steps of the algorithm are similar to the joint uplink power control and beamforming update equations. In [11], we have shown that in a feasible network, the first two iterations of Algorithm B converge to a fixed power allocation $\mathbf{P}$. Therefore the beamforming vectors are also converging to fixed beamforming vectors, which are given by

$$
\hat{\boldsymbol{w}}_{i}=\arg \max _{\boldsymbol{w}_{i}} \frac{P_{i} \boldsymbol{w}_{i}^{H} \boldsymbol{G}_{i i}^{s} \boldsymbol{w}_{i}}{\sum_{m} P_{m} \boldsymbol{w}_{i}^{H} \boldsymbol{G}_{m i}^{I} \boldsymbol{w}_{i}+\boldsymbol{w}_{i}^{H} \boldsymbol{w}_{i}} .
$$

As a result, the uplink virtual gain matrix $\boldsymbol{D}_{w}[n] \boldsymbol{F}_{w}[n]$ as well as the downlink gain matrix $\boldsymbol{D}_{w}[n] \boldsymbol{F}_{w}^{T}[n]$ converge to constant matrices. From the feasibility of the virtual uplink network, we conclude that the uplink gain matrix $\boldsymbol{D}_{w}[n] \boldsymbol{F}_{w}[n]$ converges to a matrix whose eigenvalues are inside the unit circle. The eigenvalues of uplink and downlink gain matrix are the same, as is shown in the following. The eigenvalues of $\boldsymbol{D}_{w} \boldsymbol{F}_{w}$ are the roots of $\left|\boldsymbol{D}_{w} \boldsymbol{F}_{w}-\lambda I\right|$

$$
\begin{aligned}
\left|\boldsymbol{D}_{w} \boldsymbol{F}_{w}-\lambda I\right| & =\left|\boldsymbol{D}_{w}\right|\left|\boldsymbol{F}_{w}-\lambda \boldsymbol{D}_{w}^{-1}\right|=\left|\boldsymbol{D}_{w}\right|\left|\left(\boldsymbol{F}_{w}-\lambda \boldsymbol{D}_{w}^{-1}\right)^{T}\right| \\
& =\left|\boldsymbol{D}_{w}\right|\left|\boldsymbol{F}_{w}^{T}-\lambda \boldsymbol{D}_{w}^{-1}\right|=\left|\boldsymbol{D}_{w} \boldsymbol{F}_{w}^{T}-\lambda \boldsymbol{I}\right|=0 .
\end{aligned}
$$

That is, the downlink gain matrix is an asymptotically constant matrix whose eigenvalues are inside the unit circle. Based on Theorem 1, the downlink iteration is also convergent, and the algorithm converges to a feasible solution.

From the above discussion, we can also conclude that the spectral radius of $\boldsymbol{D}_{w} \boldsymbol{F}_{w}$ and $\boldsymbol{D}_{w} \boldsymbol{F}_{w}^{T}$ are the same. Then $\rho\left(\boldsymbol{D}_{w} \boldsymbol{F}_{w}\right) \leq 1$ when $\rho\left(\boldsymbol{D}_{w} \boldsymbol{F}_{w}^{T}\right) \leq 1$ and vice versa. That is, the feasibility of virtual uplink and downlink are equivalent.

\section{B. Optimality of the Solution}

In the following we will show Algorithm B also minimizes the total transmitted power.

Theorem 2: Algorithm B minimizes the total transmitted power for the downlink, i.e.,

$$
[\hat{\boldsymbol{A}}, \hat{\boldsymbol{P}}]=\arg \min _{\boldsymbol{A}, \tilde{\boldsymbol{P}}} \sum_{i} \tilde{P}_{i}\left\|\boldsymbol{w}_{i}\right\|^{2}
$$

where $\hat{A}$ is the set of optimal beamforming vectors $\hat{A}=$ $\left\{\hat{\boldsymbol{w}}_{1}, \cdots, \hat{\boldsymbol{w}}_{M}\right\}$.

Proof: Total transmitted power can be expressed as $\mathbf{1}_{w}^{T} \tilde{\boldsymbol{P}}$, where $\mathbf{1}_{w}=\left[\left\|\boldsymbol{w}_{1}\right\|^{2}, \cdots,\left\|\boldsymbol{w}_{M}\right\|^{2}\right]^{T}$. The virtual uplink iteration converges to

$$
\begin{aligned}
\boldsymbol{P} & =\left(\boldsymbol{I}-\boldsymbol{D}_{w} \boldsymbol{F}_{w}\right)^{-1} \boldsymbol{u}_{w} \\
& =\left(\boldsymbol{I}-\boldsymbol{D}_{w} \boldsymbol{F}_{w}\right)^{-1} \boldsymbol{D}_{w} \mathbf{1}_{w} .
\end{aligned}
$$

Here we used the fact that $\boldsymbol{u}_{w}=\boldsymbol{D}_{w} \mathbf{1}_{w}$. Define $\boldsymbol{N}=$ $\left[\tilde{N}_{1}, \cdots, \tilde{N}_{M}\right]^{T}$. It follows that $\tilde{\boldsymbol{u}}_{w}=\boldsymbol{D}_{w} \boldsymbol{N}$. A weighted sum of the virtual uplink power vector defined as $\boldsymbol{P}^{T} N$ can be written as

$$
\begin{aligned}
\boldsymbol{P}^{T} \boldsymbol{N} & =\mathbf{1}_{w}^{T} \boldsymbol{D}_{w}\left(\boldsymbol{I}-\boldsymbol{D}_{w} \boldsymbol{F}_{w}^{T}\right)^{-1} \boldsymbol{N} \\
& =\mathbf{1}_{w}^{T}\left(\boldsymbol{I}-\boldsymbol{D}_{w} \boldsymbol{F}_{w}^{T}\right)^{-1} \boldsymbol{D}_{w} \boldsymbol{N} \\
& =\mathbf{1}_{w}^{T} \tilde{\boldsymbol{P}} .
\end{aligned}
$$

That is, a weighted sum of the virtual uplink power is equal to the total downlink transmitted power. Since the virtual uplink power is element-wise minimal among all power allocations [11], its weighted sum (left-hand side of the above equation) is also minimal. It can be concluded that the total transmitted power from the base stations is also minimized by Algorithm B.

By optimality, in this paper, we mean the solution that allocates the minimal power to the transmitters while the SINR at the mobile is above a target value. The objective of this work is not to minimize the transmit power for a target BER. However, if the distribution of the interference and noise is Gaussian, this algorithm also provides the optimal performance in terms of minimum BER.

In the following, using a counterexample, we will show that for the downlink there is no power allocation and beamforming 


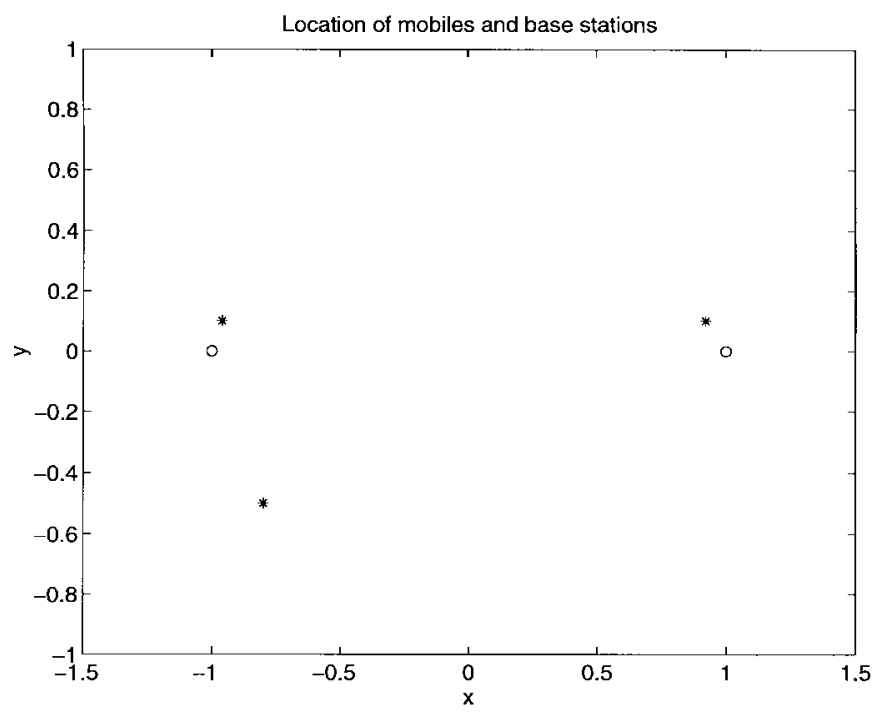

Fig. 3. Locations of mobiles and base stations (counterexample).

set that minimizes the transmitted power for each base station. We only need to find a case where there exists a feasible power allocation that transmits less power to a specific mobile than that of the minimal total power allocation. We consider a network of three mobiles and two base stations where each mobile is assigned to the closest base station as illustrated in Fig. 3. A two-element antenna is employed at each base station. The link gain between the $i$ th mobile and the $j$ th base station is simply considered to be $1 / d_{i j}^{4}$, where $d_{i j}$ is the distance between the $i$ th mobile and $j$ th base station. The noise power is assumed to be unity, and a target SINR of ten is to be achieved. The minimal power allocation by our algorithm is given by $\tilde{P}=[.6371,7.0015, .0028]^{T}$. Next, we try to reduce the interference at the first mobile by forcing the second and third beamformers to place nulls to the direction of this mobile. That is,

$$
\begin{aligned}
\boldsymbol{w}_{1}^{H}\left[\boldsymbol{a}_{11} \boldsymbol{a}_{21}\right] & =\left[\begin{array}{ll}
1 & 0
\end{array}\right], \quad \boldsymbol{w}_{2}^{H}\left[\boldsymbol{a}_{11} \boldsymbol{a}_{21}\right]=\left[\begin{array}{ll}
0 & 1
\end{array}\right], \\
\boldsymbol{w}_{3}^{H}\left[\boldsymbol{a}_{12} \boldsymbol{a}_{32}\right] & =\left[\begin{array}{ll}
0 & 1
\end{array}\right] .
\end{aligned}
$$

The weight vectors are selected such that the gain toward the desired user is unity. The weight vectors given by the above set of equations are used to construct $\boldsymbol{D}_{w}, \boldsymbol{F}_{w}$. and $\boldsymbol{u}_{w}$. The minimal power allocation to satisfy the downlink SINR requirements is then given by

$$
\tilde{\boldsymbol{P}}_{w}=\left[\boldsymbol{I}-\boldsymbol{D}_{w} \boldsymbol{F}_{w}^{T}\right]^{-1} \tilde{\boldsymbol{u}}_{w} .
$$

For the above fixed weight vectors, the minimal transmitted power by the base stations is given by $\tilde{\boldsymbol{P}}=$ $[.0316,8.4702, .036]^{T}$. Note that the transmitted power to the second and third mobiles are increased while the transmitted power to the first mobile is decreased. If there exists a feasible solution that minimizes the transmitted power for each user, it should satisfy the following condition:

$$
\hat{\boldsymbol{P}} \leq[.0316,7.0015, .0028]^{T}
$$

which contradicts with the fact that $\tilde{\boldsymbol{P}}$ minimizes the total transmitted power.

\section{Practical Implementation}

Algorithm B requires the full knowledge of the channel and array responses for the entire network. This requires channel measurements at the mobile and a feedback mechanism to send the information to the base station. Moreover, base stations should transfer the measured channel responses to the other base stations which requires a lot of wireline communication bandwidth. However, in order to calculate the transmit diversity weight vectors, we can only use the channel response to the closest cochannel users, for example the first tier in a network. In this case only the closest cochannel cells need to transfer channel responses. The practical implementation of this algorithm needs further research which is beyond the scope of this work.

In the case that the uplink and downlink are reciprocal, the virtual uplink in Algorithm B is same as the real uplink. We propose the following algorithm for reciprocal networks.

Algorithm C:

1) Diversity combining and equalization for uplink:

$$
\begin{aligned}
\boldsymbol{w}_{i}^{n} & =\arg \max _{\boldsymbol{w}_{i}} \frac{\boldsymbol{w}_{i}^{H} \Phi_{i}^{S} \boldsymbol{w}_{i}}{\boldsymbol{w}_{i}^{H} \Phi_{i}^{I} \boldsymbol{w}_{i}} \\
& =\arg \max _{\boldsymbol{w}_{i}} \frac{P_{i}^{n} \boldsymbol{w}_{i}^{H} G_{i i}^{s} \boldsymbol{w}_{i}}{\sum_{m} P_{m}^{n} \boldsymbol{w}_{i}^{H} \boldsymbol{G}_{m i}^{I} \boldsymbol{w}_{i}+N_{i} \boldsymbol{w}_{i}^{H} \boldsymbol{w}_{i}}
\end{aligned}
$$

2) Uplink power update $\left(P^{n+1}\right)$ :

$$
\boldsymbol{P}^{n+1}=\boldsymbol{D}_{w}[n] \boldsymbol{F}_{w}[n] \boldsymbol{P}^{n}+\boldsymbol{u}_{w}[n]
$$

3) Downlink power update $\left(\tilde{\boldsymbol{P}}^{n+1}\right)$ :

$$
\tilde{\boldsymbol{P}}^{n+1}=\boldsymbol{D}_{w}[n] \boldsymbol{F}_{w}^{T}[n] \tilde{\boldsymbol{P}}^{n}+\tilde{\boldsymbol{u}}_{w}[n]
$$

where $\left[\boldsymbol{u}_{w}[n]\right]_{i}=\left(\gamma_{i} N_{i} /\left(\boldsymbol{w}_{i}^{n}\right)^{H} \boldsymbol{G}_{i i}^{S} \boldsymbol{w}_{i}^{n}\right)$. The second and third steps of the algorithm can be implemented using only local interference measurements similar to any distributed power allocation algorithm [16]. In order to implement the first step of the algorithm we can use (7), where we need to estimate only the channel response to the desired user, or we can use a training sequence and use (8) to calculate $\boldsymbol{w}_{i}^{n}$. The second step of the algorithm requires the measurement of the SINR at the mobile and the link gain from each base station to its assigned mobile. In the following we will show that SINR can be estimated using the minimum mean squared error at the beamforming step $E_{\min }$. Without loss of generality, we assume that the variance of reference signal is unity. We can then show that [1]

$$
\begin{aligned}
E_{i, \min } & =\min _{\boldsymbol{w}_{i}} E\left\{|| d_{i}-\left.\boldsymbol{w}_{i}^{H} \boldsymbol{x}_{i}\right|^{2}\right\} \\
& =1-\boldsymbol{w}_{i}^{H} \Phi_{i} \boldsymbol{w}_{i}=1-P_{i} \boldsymbol{g}_{i i}^{H} \Phi_{i}^{-1} \boldsymbol{g}_{i i} .
\end{aligned}
$$

The total power at the output of the beamformer is given by

$$
P^{T}=\boldsymbol{w}_{i}^{H} \Phi_{i} \boldsymbol{w}_{i}=P_{i} \boldsymbol{g}_{i i}^{H} \Phi_{i}^{-1} \boldsymbol{g}_{i i} .
$$

Desired signal power is then expressed as

$$
\begin{aligned}
P_{i}^{s} & =P_{i} \boldsymbol{w}_{i}^{H} \boldsymbol{G}_{i i}^{s} \boldsymbol{w}_{i}=P_{i}^{2} \boldsymbol{g}_{i i}^{H} \Phi_{i}^{-1} \boldsymbol{g}_{i i} \boldsymbol{g}_{i i}^{H} \Phi_{i}^{-1} \boldsymbol{g}_{i i} \\
& =P_{i}^{2}\left(\boldsymbol{g}_{i i}^{H} \Phi_{i}^{-1} \boldsymbol{g}_{i i}\right)^{2}
\end{aligned}
$$


and the SINR at the output of beamformer can be written as

$$
\begin{aligned}
\Gamma_{i} & =\frac{P_{i}^{2}\left(\boldsymbol{g}_{i}^{H} \Phi_{i}^{-1} \boldsymbol{g}_{i i}\right)^{2}}{P_{i} \boldsymbol{g}_{i i}^{H} \Phi_{i}^{-1} \boldsymbol{g}_{i i}-P_{i}^{2}\left(\boldsymbol{g}_{i i}^{H} \Phi_{i}^{-1} \boldsymbol{g}_{i i}\right)^{2}} \\
& =\frac{P_{i}\left(\boldsymbol{g}_{i i}^{H} \Phi_{i}^{-1} \boldsymbol{g}_{i i}\right)}{1-P_{i}\left(\boldsymbol{g}_{i i}^{H} \Phi_{i}^{-1} \boldsymbol{g}_{i i}\right)}=\frac{1-E_{i, \min }}{E_{i, \min }}
\end{aligned}
$$

The power control equations in the above algorithm are then modified as

$$
P_{i}^{n+1}=P_{i}^{n} \frac{\gamma_{i}}{\Gamma_{i}}=\gamma_{i} P_{i}^{n} \frac{E_{i, \min }}{1-E_{i, \min }}
$$

Therefore, in order to update the transmitted power, $E_{\min }$ is evaluated at each base station (measured locally) and sent to the assigned mobile. Knowing its previous transmitted power and the target SINR, the mobile will update its power. The uplink weight vectors are calculated using only local measurements at each base station. That is, this algorithm is amenable to a distributed implementation. In this case there is no need to perform the global measurements to find the channel responses. Note that the only difference between this algorithm and Algorithm B is that the channel response for both uplink and downlink are the same, and additive noise at the mobile is contributing in the uplink beamforming and power allocation. Using similar proof as in Section IV-A, we can show that Algorithm $\mathrm{C}$ converges to a feasible solution for the downlink, and the total power is minimized if the additive noise at all mobiles is the same.

In any of the above cases, the downlink power iteration can be implemented using only local downlink measurements at mobile. Moreover, we can replace the first two steps of the algorithm with different beamforming schemes which may or may not require global channel measurements. When we find the beamforming vectors, we can calculate the minimal power allocation for the downlink using the third step of the algorithm. In the next section, for comparison purposes we compare the performance of our algorithms with a modified version of the algorithms proposed in [7]. In the first algorithm we try to maximize the received power at the desired mobile with a fixed norm transmit beamforming vector.

Algorithm D:

1) At each base station:

$$
\begin{aligned}
& \boldsymbol{w}_{i}=\arg \max _{\boldsymbol{w}} \boldsymbol{w}^{H} \boldsymbol{G}_{i i}^{s} \boldsymbol{w}, \\
& \text { subject to }\|\boldsymbol{w}\|^{2}=c
\end{aligned}
$$

2) Power allocation:

$$
\tilde{\boldsymbol{P}}=\left[\boldsymbol{I}-\boldsymbol{D}_{w} \boldsymbol{F}_{w}^{T}\right]^{-1} \tilde{\boldsymbol{u}}_{w}
$$

Note that the magnitude of the weight vectors $c$ does not change the end result, since the power allocation step will adjust the overall magnitude of the transmit power. The solution to the beamforming is the principal eigenvector of $\boldsymbol{G}_{i i}^{s}$. In another algorithm we try to maximize the gain toward the desired user while the total transmitted power to all other users is minimized.

\section{Algorithm E:}

1) At each base station:

$$
\begin{aligned}
& \boldsymbol{w}_{i}=\arg \max _{\boldsymbol{w}} \boldsymbol{w}^{H} \boldsymbol{G}_{i i}^{\boldsymbol{s}} \boldsymbol{w} \\
& \text { subject to } \boldsymbol{w}^{H}\left(\sum_{j} \boldsymbol{G}_{j i}^{I}\right) \boldsymbol{w}<c .
\end{aligned}
$$

2) Power allocation:

$$
\tilde{\boldsymbol{P}}=\left[\boldsymbol{I}-\boldsymbol{D}_{w} \boldsymbol{F}_{w}^{T}\right]^{-1} \tilde{\boldsymbol{u}}_{w}
$$

where $c$ is a constant and does not affect the final result. The solution to the beamforming is the generalized eigenvector of $\left[\boldsymbol{G}_{i i}^{s}, \Sigma_{j} \boldsymbol{G}_{i j}^{I}\right]$. In Algorithms D and E the first step determines the beamforming vector. The second step adjusts the norm of the weigh vectors or the transmit power to set the SINR at each link to the target value. We have modified the algorithm proposed in [9] for the case where adaptive arrays are only used at the transmitters. In this algorithm, the weight vectors calculated from the uplink are used for transmit beamforming. Then, the minimal power allocation for the downlink is found by the downlink power control.

\section{Algorithm F:}

1)

$$
\hat{\boldsymbol{w}}_{i}=\arg \max _{\boldsymbol{w}_{i}} \frac{\boldsymbol{w}_{i}^{H} \boldsymbol{\Phi}_{i}^{s} \boldsymbol{w}_{i}}{\boldsymbol{w}_{i}^{H} \Phi_{i}^{I} \boldsymbol{w}_{i}}
$$

2)

$$
\tilde{\boldsymbol{P}}=\left[\boldsymbol{I}-\boldsymbol{D}_{w} \boldsymbol{F}_{w}^{T}\right]^{-1} \tilde{\boldsymbol{u}}_{w}
$$

In the simulation study we will evaluate the maximum achievable SINR using Algorithms $\mathrm{C}$ and $\mathrm{F}$ in a TDD network. We will also evaluate the performance of the above algorithms when we use the uplink channel response as an estimate of the downlink channel response.

\section{Simulation Results}

In order to evaluate the performance of our algorithms, a network with hexagonal cells and a cluster size of one is simulated as illustrated in Fig. 4. The base stations are placed at the center of the cell. In each cell one user is placed randomly with a uniform distribution. The path loss is proportional to $r^{-4}$, where $r$ is the distance between the mobile and base station. For each link, 3-dB log-normal shadow fading and three paths with equal power Rayleigh fading are considered. In the first simulation we considered negligible delay spread between different paths. The angle of arrival for each path is a uniform random variable in $[0,2 \pi]$. Raised cosine function is used as the pulse shaping function. With different numbers of antennas at the base stations we have implemented Algorithms B-E and evaluated the maximum achievable SINR in the network. Algorithms D and E with fixed power allocation (without power control) are called R1 and R2, respectively [7], and are simulated in a system where each base station transmits with the maximum power. The results of a Monte Carlo analysis for 100 iterations is summarized in Table I. In the same system with single antennas at the base stations, 


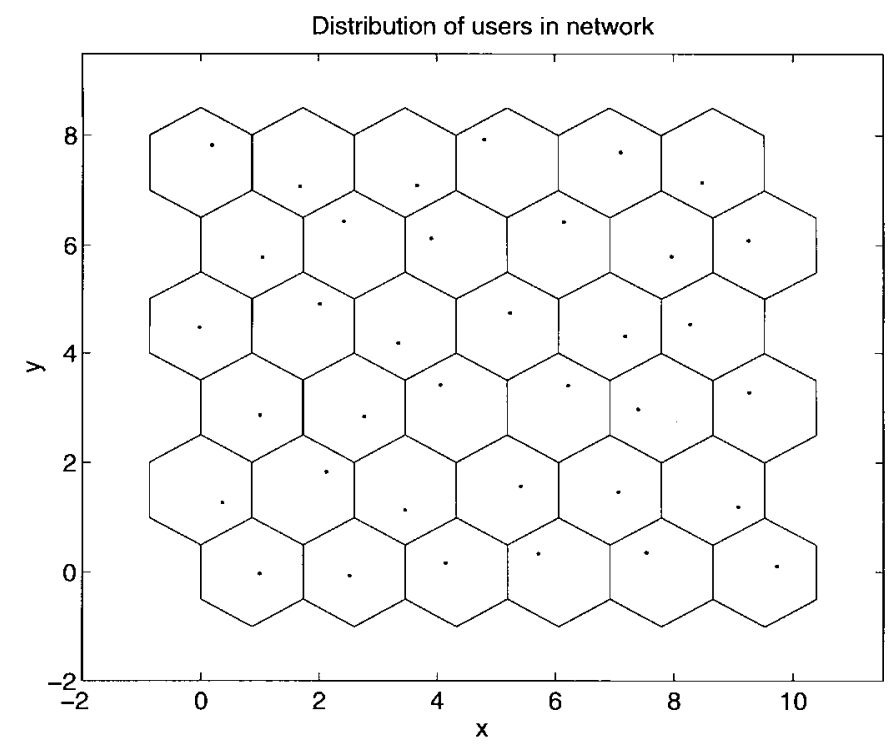

Fig. 4. Locations of mobiles and base stations.

TABLE I

MaXimum Achievable SINR in dB

\begin{tabular}{c|c|c|c|c}
\hline Number of elements & 2 & 4 & 6 & 8 \\
\hline Algorithm B & 9.14 & 12.5 & 14.8 & 16.7 \\
\hline Algorithm D & 7.5 & 9.6 & 10.9 & 11.74 \\
\hline Algorithm E & 6.9 & 7.9 & 8.4 & 8.3 \\
\hline Algorithm R1 & -4.8 & -2.9 & -1.8 & -.95 \\
\hline Algorithm R2 & -6.3 & -5.0 & -4.4 & -4.5 \\
\hline
\end{tabular}

TABLE II

MaXimum ACHIEVAbLe SINR IN dB

\begin{tabular}{c|c|c|c|c}
\hline Number of elements & 2 & 4 & 6 & 8 \\
\hline Algorithm B & 7.5 & 9.08 & 9.64 & 9.9 \\
\hline Algorithm D & 7.1 & 9.3 & 10.2 & 10.6 \\
\hline Algorithm E & 6.6 & 7.1 & 6.6 & 5.0 \\
\hline Algorithm R1 & -4.7 & -2.8 & -2.05 & -1.7 \\
\hline Algorithm R2 & -5.6 & -4.6 & -5.9 & -7.7 \\
\hline
\end{tabular}

the optimal power control algorithm will achieve maximum SINR of $5.2 \mathrm{~dB}$. From the simulation results we conclude that our optimal algorithm can achieve considerably higher SINR. By adding the power allocation to R1 and R2 we can improve the SINR significantly. Algorithm D uses a simpler beamforming algorithm and requires the channel response from each base station to only its desired user. Note that in all of the above simulations the downlink channel response from each base station to each mobile is assumed to be known. The same simulation is performed where in the beamforming step the uplink channel responses are used as an estimation of the downlink channel responses. In this system, the uplink frequency is $10 \%$ lower than that of the downlink. The result is summarized in Table II. The performance is degraded compared to the previous case.

Algorithms $\mathrm{C}$ and $\mathrm{F}$ are designed for reciprocal networks. We have evaluated the performance of these algorithms in a TDD network with the same system setup as in the previous case. The uplink and downlink channel responses are assumed to be reciprocal. The result of Monte Carlo simulations for the
TABLE III

MAXIMUM ACHIEVABLE SINR IN dB

\begin{tabular}{c|c|c|c|c}
\hline Number of elements & 2 & 4 & 6 & 8 \\
\hline Algorithm C & 9.0 & 12.6 & $\mathbf{1 4 . 9}$ & 16.9 \\
\hline Algorithm F & 8.1 & 11.05 & 12.9 & 14.5 \\
\hline
\end{tabular}

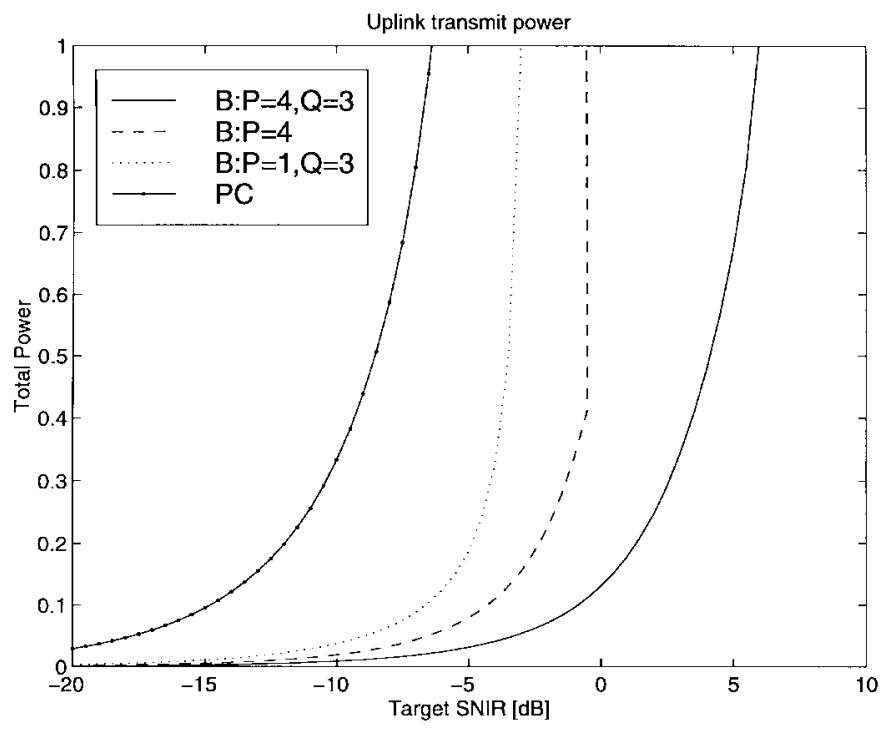

Fig. 5. Total mobile power as a function of target SINR.

maximum achievable SINR for those algorithms are summarized in Table III. Note the differences between Algorithms C and F. In Algorithm F, the beamforming vectors are evaluated once, while in Algorithm $\mathrm{C}$ the beamforming vectors are adjusted after each power control step.

In the same cellular network as in Fig. 4, we have simulated a system where base stations are equipped with four-element antenna arrays. Channel responses are constructed as before. However, the delay spread in the channel is considered to be uniformly distributed in $[0,4 T]$, where $T$ is the symbol interval. The multipath fading and angles of arrival are also independent in uplink and downlink. We consider an FDD system with $10 \%$ difference between uplink and downlink frequencies. The total transmitted power in uplink and downlink as a function of the target SINR is shown in Figs. 5 and 6, respectively. In Fig. 5 the total uplink power as a function of the target SINR is plotted. The dashed curve shows the case where we use single tap diversity combiner and four-element antennas at the receivers in the uplink. The solid curve shows the total transmitted power when we use three-tap receiver diversity combiners and four-element antenna arrays. The results are also compared with the case where we use omnidirectional antennas and power control without equalization (PC: $Q=1$ ), and with three tap equalizer (PC: $P=1, Q=3$ ). Fig. 6 shows the total downlink transmitted power as a function of the target SINR at the mobile. In this simulation we have used four element antennas at the base station. Performance of algorithm B with single tap and multitap transmit diversity $(Q=3)$ are compared to Algorithms D, E, R1, and R2. The curve labeled with PC shows the result of a power-controlled system with single antennas. The simulation results show that by using our algorithms, we can significantly reduce the total 


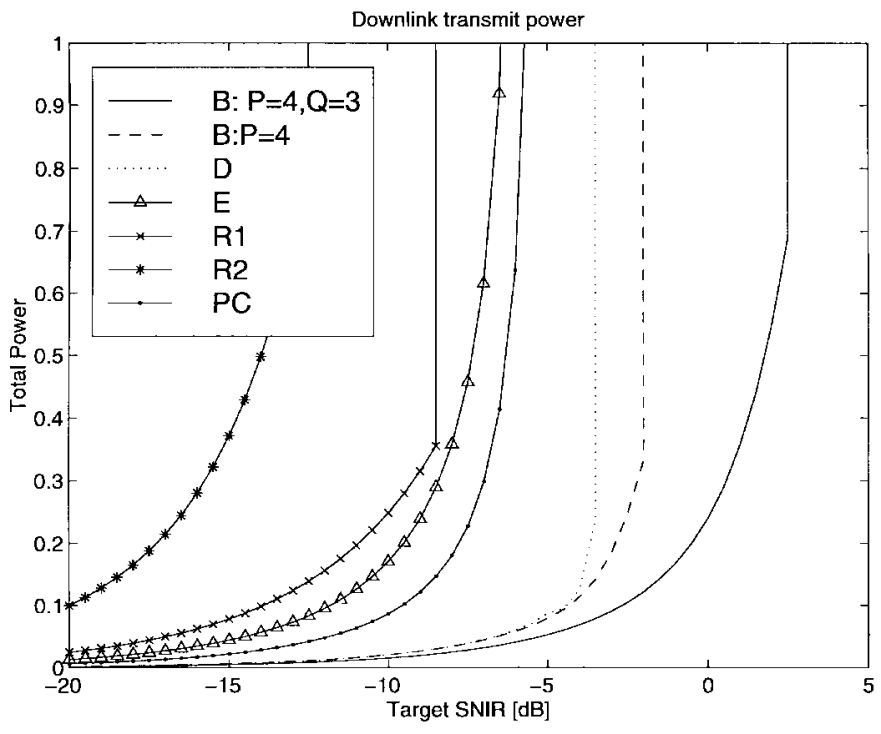

Fig. 6. Total base station power as a function of target SINR.

transmitted power, or maximum achievable SINR, in both uplink and downlink. We can also increase the SINR for a given transmitted power.

\section{CONCLUSION}

We have proposed an algorithm for transmit beamforming jointly with power control, which achieves a feasible set of transmit beamforming vectors if there exist any. It also minimizes the total transmitted power in a network for a target SINR at each mobile. The solution proposed in this work is also extended to multitap transmit beamformers. We have shown that our schemes can reduce the total transmitted power compared to previously proposed methods. They can also increase the maximum number of allowable users or maximum achievable SINR in a network. In TDD networks where the uplink and downlink channels are reciprocal, this algorithm can be implemented using only local measurements and in a distributed fashion without any performance degradation. In the networks where uplink and downlink channels are different due to FDD or fading, in order to utilize the full capacity of the network, the algorithm shall be implemented in a centralized fashion, using downlink channel responses.

\section{APPENDIX}

\section{A. Multitap Receiver Diversity Combiner}

In a single tap diversity combiner, the delayed version of the signal of interest is considered as interference, which is rejected at the output of the combiner. While in a multitap combiner the desired signal and its delayed versions can contribute to the estimation of the transmitted symbol (Fig. 7). In a sense, the multitap diversity combiner is a broadband beamformer, and the simple combiner is a narrowband beamformer. It is clear that the broadband one performs better in multipath environments where the delay spread of signals produces effective broadband signals. The $T$ blocks in Fig. 7 produce one symbol interval delay, and the combiner simply

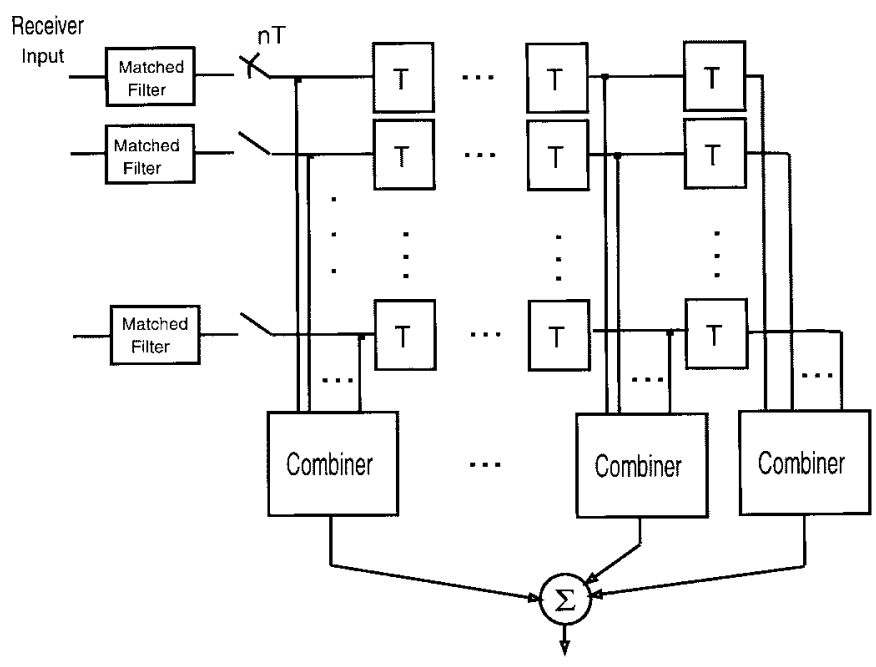

(a)

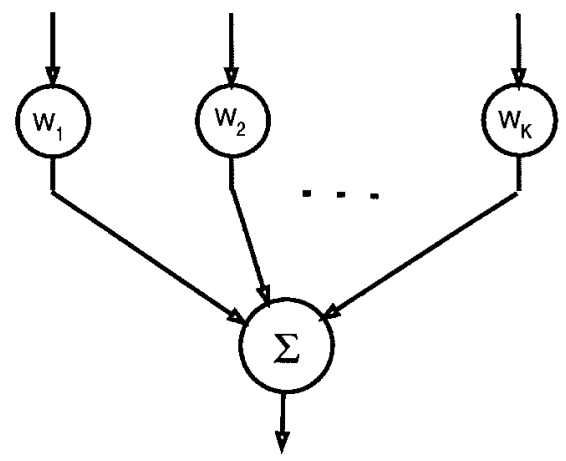

(b)

Fig. 7. (a) Block diagram of a multitap receiver diversity system; (b) a combiner.

calculates the weighted sum of its input signals. The maximum delay of the diversity combiner, denoted by $Q$, is also called the length of equalizer. The output of the diversity combiner can be expressed as

$$
y_{i}(k)=\sum_{q=0}^{Q-1} \boldsymbol{w}_{i}^{H}(q) \boldsymbol{x}_{i}(k+q) .
$$

To simplify the derivations, we define $\boldsymbol{W}_{\boldsymbol{i}}=\left[\boldsymbol{w}_{i}^{T}(0), \cdots\right.$, $\left.\boldsymbol{w}_{i}^{T}(Q-1)\right]^{T}$ and $\boldsymbol{X}_{i}(k)=\left[\boldsymbol{x}_{i}^{T}(k), \cdots, \boldsymbol{x}_{i}^{T}(k+Q-1)\right]^{T}$. The diversity combining output is then given by

$$
y_{i}(k)=\boldsymbol{W}_{i}^{H} \boldsymbol{X}_{i}(k) .
$$

In the combining process we try to minimize the difference of the output of combiner $\left\{y_{i}(k)\right\}$ and the training sequence $\left\{d_{i}(k)\right\}$

$$
E_{i, \min }=\min _{\boldsymbol{W}_{i}} E\left\{\left|d_{i}(k)-\boldsymbol{W}_{i}^{H} \boldsymbol{X}_{i}(k)\right|^{2}\right\} .
$$

The combining vector $W_{i}$ is given by

$$
W_{i, \min }=\arg \min _{W_{i}} E\left\{\left|d_{i}(k)-W_{i}^{H} \boldsymbol{X}_{i}(k)\right|^{2}\right\} .
$$

The solution is given by

$$
W_{i, \min }=\Phi_{i}^{-1} p_{i}
$$


where

$$
\Phi_{i}=E\left(X_{i}(k) X_{i}^{H}(k)\right)
$$

and

$$
\boldsymbol{p}_{i}=E\left(d_{i}^{*}(k) \boldsymbol{X}_{i}(k)\right)
$$

Assume the training sequence is a delayed version of the transmitted signal, i.e., $d_{i}=s_{i}(k+D)$, where $D$ is chosen to center the space time combiner, i.e., $D=Q / 2$ and $Q>N$. The cross correlation vector $\boldsymbol{p}_{i}$ is given by

$$
\boldsymbol{p}_{i}=E\left\{s_{i}(k+Q / 2) \boldsymbol{X}_{i}\right\}=\sqrt{P_{i}}\left[\begin{array}{c}
\mathbf{0} \\
\boldsymbol{h}_{i i}(0) \\
\vdots \\
\boldsymbol{h}_{i i}(N-1) \\
\mathbf{0}
\end{array}\right]=\sqrt{P_{i}} \boldsymbol{g}_{i i} .
$$

The noise vector $n_{i}$ consists of spatially and temporally white noise components which are independent of the received signal. Therefore, the correlation matrix can be simplified as

$$
\begin{aligned}
\Phi_{i}= & \sum_{m=1}^{M} P_{m} E\left\{\left[\begin{array}{c}
\boldsymbol{H}_{m i} \boldsymbol{s}_{m}(k) \\
\vdots \\
\boldsymbol{H}_{m i} \boldsymbol{s}_{m}(k+Q-1)
\end{array}\right]\right. \\
& \left.\cdot\left[\boldsymbol{s}_{m}^{H}(k) \boldsymbol{H}_{m i}^{H} \quad \cdots \quad \boldsymbol{s}_{m}^{H}(k+Q-1) \boldsymbol{H}_{m i}^{H}\right]\right\}+N_{i} \boldsymbol{I}
\end{aligned}
$$

where $N_{i}$ is the thermal noise power at the input of each array element. We assume the signals transmitted from different sources are uncorrelated, and the signal transmitted from a source is also an uncorrelated zero mean sequence of symbols. Then (19) can be simplified as

$$
\Phi_{i}=\sum_{m=1}^{M} P_{m} G_{m i}+N_{i} \boldsymbol{I}
$$

$\boldsymbol{G}_{m i}$ is a block matrix whose $p q$ th block is defined as

$$
\left[\boldsymbol{G}_{m i}\right]_{p q}=\boldsymbol{H}_{m i} \boldsymbol{J}_{p-q} \boldsymbol{H}_{m i}^{H}
$$

where $\boldsymbol{J}_{p-q}$ is a matrix which only has all ones on the $(p-q)$ th diagonal in parallel with main diagonal elements. Note that the formulation of the correlation matrix, and consequently, the multitap diversity combiner SINR, are similar to that of the single tap diversity combiner. As a result, Algorithm A can be used to find the optimal power allocation and receiver beamforming vectors.

\section{B. Multitap Transmit Diversity}

The block diagram of a multitap transmit diversity system is shown in Fig. 8, which is the dual of the graph in Fig. 7. The transmitted signal is a combination of the desired signal and its delayed versions. Denote the diversity vector at the $q$ th

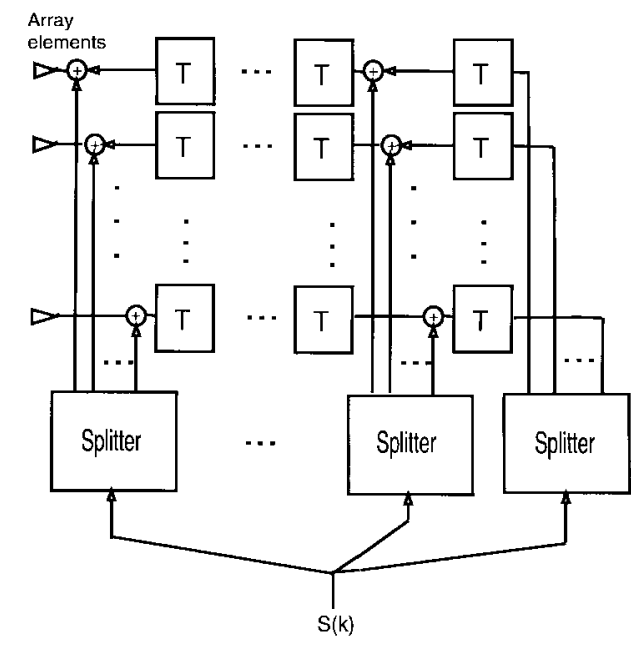

Fig. 8. Block diagram of a multitap transmit diversity system.

tap of the $b$ th base station by $w_{b}(q)$. The received signal at the $i$ th mobile is given by

$$
\begin{aligned}
\tilde{y}_{i}(t)= & \sum_{b=1}^{B} \sum_{q=0}^{Q-1} \sum_{n=0}^{k} \sum_{l=1}^{L} \boldsymbol{w}_{b}^{H}(q) \boldsymbol{a}_{i b}\left(\theta_{l}\right) \sqrt{\rho_{i b} G_{i b} \alpha_{i b}^{l}} g_{i b} \\
& \cdot\left(t-n T-\tau_{i b}^{l}\right) \sqrt{\tilde{P}_{b}} \tilde{s}_{b}(n-q)+\tilde{n}_{i}(t) .
\end{aligned}
$$

The sampled signal at the output of the receiver filter is given by

$\tilde{z}_{i}(k)=\sum_{b=1}^{B} \sum_{q=0}^{Q-1} \sum_{n=0}^{N-1} \boldsymbol{w}_{b}^{H}(q) \boldsymbol{h}_{i b}(n) \sqrt{\tilde{P}_{b}} \tilde{s}_{b}(k-n+q)+\tilde{n}_{i}(k)$

where $\boldsymbol{h}_{i b}$ is defined as in the signal tap diversity combiner. It follows that

$$
\tilde{z}_{i}(k)=\sum_{b=1}^{B} \sum_{q=0}^{Q-1} \boldsymbol{w}_{b}^{H}(q) \boldsymbol{H}_{i b} \sqrt{\tilde{P}_{b}} \tilde{\boldsymbol{s}}_{b}(k+q)+\tilde{n}_{i}(k)
$$

where $\tilde{\boldsymbol{s}}_{b}(k)=\left[\tilde{s}_{b}(k), \cdots, \tilde{s}_{b}(k-N+1)\right]^{T}$. Define $\boldsymbol{W}_{b}=$ $\left[\boldsymbol{w}_{b}^{T}(0), \cdots, \boldsymbol{w}_{b}^{T}(Q-1)\right]^{T}$, and $\tilde{\boldsymbol{X}}_{i b}(k)=\left[\tilde{\boldsymbol{x}}_{i b}^{T}(k), \cdots, \tilde{\boldsymbol{x}}_{i b}^{T}(k+\right.$ $Q-1)]^{T}$, where $\tilde{\boldsymbol{x}}_{i b}(k)=\boldsymbol{H}_{i b} \sqrt{\tilde{P}_{b}} \widetilde{\boldsymbol{s}}(k)$. Then the received signal at the $i$ th receiver is expressed as

$$
\tilde{z}_{i}(k)=\sum_{b} W_{b}^{H} \tilde{\boldsymbol{X}}_{i b}(k)+\tilde{n}_{i}(k) .
$$

The total transmitted downlink power by each transmitter is given by

$$
\sum_{q=0}^{Q-1} \tilde{P}_{i}\left(\boldsymbol{w}_{i}^{n}\right)^{H}(q) \boldsymbol{w}_{i}^{n}(q)=\tilde{P}_{i}\left(\boldsymbol{W}_{i}^{n}\right)^{H} \boldsymbol{W}_{i}^{n}
$$

Again the expression for the received signal is similar to that of the single tap transmit diversity system, where $x_{i b}$ is replaced with $\boldsymbol{X}_{i b}$, and $\boldsymbol{w}_{i}$ is replaced with $\boldsymbol{W}_{i}$. Therefore, Algorithm B can be applied to multitap diversity systems as well. 


\section{Oversampling Method}

Instead of sampling the matched filter output, we can oversample the received vector at the array output by a factor of $R$. In this case the samples are taken at $n T / R$ where $n \in Z$. The block diagram of the system is similar to Fig. 7 , where the delay blocks $T$ are replaced by $T / R$. The received signal is given by

$$
\begin{aligned}
\boldsymbol{x}_{i}(t)= & \sum_{m=1}^{M} \sum_{n=0}^{k} \sum_{l=1}^{L} \sqrt{\rho_{m i} G_{m i} \alpha_{m i}^{l} P_{m}} \boldsymbol{a}_{m i}\left(\theta_{l}\right) \\
& \cdot g_{m i}\left(t-n T-\tau_{m i}^{l}\right) s_{m}(n)+\boldsymbol{n}_{i}(t)
\end{aligned}
$$

and the sampled signal is given by

$$
\begin{aligned}
\boldsymbol{x}_{i}(k T+ & r T / R) \\
= & \sum_{m=1}^{M} \sum_{n=0}^{k} \sum_{l=1}^{L} \sqrt{\rho_{m i} G_{m i} \alpha_{m i}^{l} P_{m}} a_{m i}\left(\theta_{l}\right) \\
& \cdot g_{m i}\left(k T+r T / R-n T-\tau_{m i}^{l}\right) s_{m}(n) \\
& +\boldsymbol{n}_{i}(k T+r T / R), \quad r=0, \cdots, R-1 .
\end{aligned}
$$

Define

$$
\boldsymbol{h}_{m i}(t)=\sum_{l=1}^{L} \boldsymbol{a}_{m i}\left(\theta_{l}\right) \sqrt{\rho_{m i} G_{m i} \alpha_{m i}^{l}} g_{m i}\left(t-\tau_{m i}^{l}\right) .
$$

The sampled received signal is then given by

$$
\begin{aligned}
\boldsymbol{x}_{i}(k T+ & r T / R) \\
= & \sum_{m=1}^{M} \sum_{n=0}^{k} \sqrt{P_{m}} \boldsymbol{h}_{m i}((k-n) T+r T / R) s_{m}(n) \\
& +\boldsymbol{n}_{i}(k T+r T / R) \\
= & \sum_{m=1}^{M} \sum_{n=0}^{k} \sqrt{P_{m}} \boldsymbol{h}_{m i}(n T+r T / R) \\
& \cdot s_{m}(k-n)+\boldsymbol{n}_{i}(k T+r T / R) .
\end{aligned}
$$

Assume that the length of the impulse response is $N$. Then the received signal can be expressed as

$$
\begin{aligned}
\boldsymbol{x}_{i}(k T+ & +r T / R)=\sum_{m=1}^{M} \sqrt{P_{m}} \boldsymbol{H}_{m i}(r T / R) \boldsymbol{s}_{m}(k) \\
& +\boldsymbol{n}_{i}(k T+r T / R), \quad r=0, \cdots, R-1
\end{aligned}
$$

where

$$
\boldsymbol{H}_{m i}(r T / R)=\left[\boldsymbol{h}_{m i}(r T / R), \cdots, \boldsymbol{h}_{m i}((N-1) T+r T / R)\right]
$$

and

$$
\boldsymbol{s}_{m}(k)=\left[s_{m}(k), \cdots, s_{m}(k-N+1)\right]^{T} .
$$

Define a received vector $\boldsymbol{X}_{i}(k)$ which consists of the received vectors at the sampling points

$$
\begin{aligned}
& \boldsymbol{X}_{i}(k) \\
& =\left[\boldsymbol{x}_{i}(k T) \quad \boldsymbol{x}_{i}(k T+T / R), \cdots, \boldsymbol{x}_{i}(k T+(R-1) T / R)\right]^{T} .
\end{aligned}
$$

From (24) and (25) it follows that

$$
\begin{aligned}
& {\left[\begin{array}{c}
\boldsymbol{x}_{i}(k T) \\
\vdots \\
\boldsymbol{x}_{i}(k T+(R-1) T / R)
\end{array}\right]} \\
& =\sum_{m}\left[\begin{array}{c}
\boldsymbol{H}_{m i}(r T / R) \\
\vdots \\
\boldsymbol{H}_{m i}((R-1) T / R)
\end{array}\right] \boldsymbol{s}_{m}(k) \\
& +\left[\begin{array}{c}
\boldsymbol{n}_{i}(k T) \\
\vdots \\
\boldsymbol{n}_{i}(k T+(R-1) T / R)
\end{array}\right]
\end{aligned}
$$

or

$$
\boldsymbol{X}_{i}(k)=\sum_{m} \mathcal{H}_{m i} \sqrt{P_{m}} \boldsymbol{s}_{m}(k)+\mathcal{N}_{i}(k)
$$

where

$$
\mathcal{H}_{m i}=\left[\begin{array}{c}
\boldsymbol{H}_{m i}(r T / R) \\
\vdots \\
\boldsymbol{H}_{m i}((R-1) T / R)
\end{array}\right]
$$

and

$$
\mathcal{N}_{i}(k)=\left[\begin{array}{c}
\boldsymbol{n}_{i}(k T) \\
\vdots \\
\boldsymbol{n}_{i}(k T+(R-1) T / R)
\end{array}\right] .
$$

The output of the combiner can be written as

$$
y_{i}(k)=\sum_{q=0}^{Q-1} \boldsymbol{w}_{i}^{H}(q) \boldsymbol{X}_{i}(k+q) .
$$

Since (18) and (26) have the same structure, the formulation of this problem would be the same as before.

In the transmit diversity case, similar to the receive diversity case, we can oversample the received signal. The block diagram of the transmit diversity system would be similar to that of Fig. 8, while the delay at each tap is $T / R$. The received signal at the $i$ th mobile is given by

$$
\begin{aligned}
\tilde{y}_{i}(t)= & \sum_{b=1}^{B} \sum_{q=0}^{Q-1} \sum_{n=0}^{k} \sum_{l=1}^{L} \boldsymbol{w}_{b}^{H}(q) \boldsymbol{a}_{i b}\left(\theta_{l}\right) \sqrt{\rho_{i b} G_{i b} \alpha_{i b}^{l}} g_{i b} \\
& \cdot\left(t-n T-\tau_{i b}^{l}\right) \sqrt{P_{b}} \tilde{s}_{b}(n+q)+\tilde{n}_{i}(t)
\end{aligned}
$$

where $k=\lfloor t / T\rfloor$. Define

$$
\boldsymbol{h}_{i b}(n)=\sum_{l=1}^{L} \boldsymbol{a}_{i b}\left(\theta_{l}\right) \sqrt{\rho_{i b} G_{i b} \alpha_{i b}^{l}} g_{i b}\left(t-\tau_{i b}^{l}\right) .
$$

Then (27) can be rewritten as

$$
\begin{aligned}
\tilde{y}_{i}(t)= & \sum_{b=1}^{B} \sum_{q=0}^{Q-1} \sum_{n=0}^{k} \sum_{l=1}^{L} \boldsymbol{w}_{b}^{H}(q) \boldsymbol{a}_{i b}\left(\theta_{l}\right) \sqrt{\rho_{i b} G_{i b} \alpha_{i b}^{l}} g_{i b} \\
& \cdot\left(t-n T-\tau_{i b}^{l}\right) \sqrt{P_{b}} \tilde{s}_{b}(n-q)+\tilde{n}_{i}(t) .
\end{aligned}
$$


When the length of the channel response is less than $N$, the oversampled received signal is given by

$$
\begin{aligned}
\tilde{y}_{i}(k T+ & r T / R) \\
= & \sum_{b=1}^{B} \sum_{q=0}^{Q-1} \sum_{n=0}^{k} \boldsymbol{w}_{b}^{H}(r, q) \boldsymbol{h}_{i b}(k T+r T / R-n T) \\
& \cdot \sqrt{P_{b}} \tilde{s}_{b}(n+q)+\tilde{n}_{i}(t) \\
= & \sum_{b=1}^{B} \sum_{q=0}^{Q-1} \sum_{n=0}^{N-1} w_{b}^{H}(r, q) \boldsymbol{h}_{i b}(n T+r T / R) \\
& \cdot \sqrt{P_{b}} \tilde{s}_{b}(k-n+q)+\tilde{n}_{i}(k T+r T / R) \\
= & \sum_{b=1}^{B} \sum_{q=0}^{Q-1} \boldsymbol{w}_{b}^{H}(r, q) \boldsymbol{H}_{i b}(r T / R) \sqrt{P_{b}} \tilde{s}_{b}(k+q) \\
& +\tilde{n}_{i}(k T+r T / R)
\end{aligned}
$$

where $\boldsymbol{H}_{i b}(r T / R)$ is defined as before

$$
\boldsymbol{H}_{i b}(r T / R)=\left[h_{i b}(r T / R), \cdots, h_{i b}((N-1) T+r T / R)\right] .
$$

Define

$$
W_{b}(r)=\left[\begin{array}{c}
\boldsymbol{w}_{b}(r, 0) \\
\vdots \\
\boldsymbol{w}_{b}(r, Q-1)
\end{array}\right]
$$

and

$$
\tilde{\boldsymbol{X}}_{i b}(r T / R, k)=\left[\begin{array}{c}
\tilde{\boldsymbol{x}}_{i b}(r T / R, k) \\
\vdots \\
\tilde{\boldsymbol{x}}_{i b}(r T / R, k-Q+1)
\end{array}\right]
$$

where $\tilde{\boldsymbol{x}}_{i b}(r T / R, k)=\boldsymbol{H}_{i b}(r T / R) \sqrt{P_{b}} \tilde{\boldsymbol{s}}_{b}(k)$. Then the received signal at the $i$ th receiver is represented by

$$
\begin{aligned}
\tilde{y}_{i}(k T+r T / R)= & \sum_{b} W_{b}^{H} \tilde{\boldsymbol{X}}_{i b}(r T / R, k) \\
& +\tilde{n}_{i}(k T+r T / R) .
\end{aligned}
$$

In order to make the decision, we average the received samples, i.e.,

$$
\begin{aligned}
\tilde{z}_{i}(k) & =\sum_{r} \tilde{y}_{i}(k T+r T / R) \\
& =\sum_{r} \sum_{b} W_{b}^{H}(r) \tilde{X}_{i b}(r T / R, k)+\sum_{r} \tilde{n}_{i}(k T+r T / R) \\
& =\sum_{b} \mathcal{W}_{b}^{H} \tilde{\mathcal{X}}_{i b}(k)+\mathcal{N}_{i}(k)
\end{aligned}
$$

where

$$
\mathcal{W}_{b}^{T}=\left[W_{b}^{T}(0), \cdots, W_{b}^{T}((R-1) / R)\right]
$$

and

$$
\tilde{\mathcal{X}}_{i b}^{T}(k)=\left[\tilde{\boldsymbol{X}}_{i b}(0, k), \cdots, \tilde{\boldsymbol{X}}_{i b}(R-1) T / R, k\right) .
$$

Since (29) is similar to (21), we can calculate the multitap transmit diversity coefficients and power allocations as before.

\section{REFERENCES}

[1] R. A. Monzingo and T. W. Miller, Introduction to Adaptive Arrays. New York: Wiley, 1980.

[2] B. Suard, A. Naguib, G. Xu, and A. Paulraj, "Performance analysis of CDMA mobile communication systems using antenna array," in Proc. ICASSP '93, vol. IV, Minneapolis, MN, Apr. 1993, pp. 153-156.
[3] P. Balaban, and J. Sulz, "Optimum combining and equalization in digital data transmission with application to cellular mobile radio," IEEE Trans. Veh. Technol., pp. 342-354, May 1991.

[4] D. Gerlach and A. Paulraj, "Adaptive transmitting antenna array with feedback," IEEE Signal Processing Lett., vol. 1, no. 10, Oct. 1994.

[5] _ "Spectral reuse using transmit antenna array and feedback," in Proc. Int. Conf. on Acoustic, Speech and Signal Processing, Adelite, Australia, Apr. 1994, pp. 97-100.

[6] H. Liu and G. Xu, "Multiuser blind channel estimation and spatial channel pre-equalization," in Proc. ICASSP'95, Detroit, MI, May 1995, pp. 1756-1759.

[7] G. G. Raleigh, S. D. Diggavi, V. K. Jones, and A. Paulraj, "A blind adaptive transmit antenna algorithm for wireless communications," in Proc. ICC'95, vol. 3, p. 1949, 1995.

[8] P. Mongensen, F. Frederiskson, J. Wigard, and S. Peterson, "A research study of antenna diversity and data receivers for DECT," in Proc. Nordic Radio Symp., Slatsjobaden, Sweden, Apr. 1995.

[9] E. Perahia and G. Pottie, "Adaptive antenna arrays and equalization for indoor digital radio," in IEEE Int. Conf. Communications ICC-96, June 1996.

[10] D. Gerlach and A. Paulraj, "Adaptive transmitting antenna methods for multipath environments," in Proc. GLOBECOM'94, vol. I, pp. 425-429.

[11] F. Rashid-Farrokhi, L. Tassiulas, and K. J. R. Liu, "Joint optimal power control and beamforming in wireless networks using antenna arrays," IEEE Trans. Commun., vol. 46, pp. 1313-1324, Nov. 1998

[12] _ "Joint optimal power control and beamforming for wireless networks with antenna arrays," in Proc. IEEE Global Communications Conf., London, Nov. 1996, pp. I-555-559.

[13] A. Paulraj and C. B. Papadias, "Array processing for mobile communications," in Handbook on Signal Processing. Boca Raton, FL: CRC Press, 1996.

[14] J. H. Winters, J. Salz, and R. D. Gitlin, "The impact of antenna diversity on the capacity of wireless communication system," IEEE Trans. Commun., vol. 42, pp. 1740-1751, Feb./Mar./Apr. 1994

[15] J. L. Willems, Stability Theory of Dynamical Systems. New York: Wiley, 1970

[16] J. Zander, "Distributed cochannel interference control in cellular radio systems," IEEE Trans. Veh. Technol., vol. 41, pp. 306-311, Aug. 1992.

[17] J. H. Winters, "The diversity gain of transmit diversity in wireless systems with Rayleigh fading," in ICC 94, vol. 2, pp. 1121-1125.

[18] B. Widrow and S. Stearns, Adaptive Signal Processing. Englewood Cliffs, NJ: Prentice Hall, 1985.

[19] S. J. Orfanidis, Optimal Signal Processing: An Introduction. New York: Macmillan, 1985.

[20] F. Rashid-Farrokhi, K. J. R. Liu, and L. Tassiulas, "Transmit beamforming for cellular wireless communications," in Proc. 31st Annu. Conf. Information Sciences and Systems, (CISS-97), Baltimore, vol. 1, Mar. 1997, pp. 92-97.

[21] _ "Transmit and receive diversity and equalization in wireless networks with fading channels," in Proc. GLOBECOM'97, Phoenix, AZ, Nov. 1997, vol. 3, pp. 1193-1198.

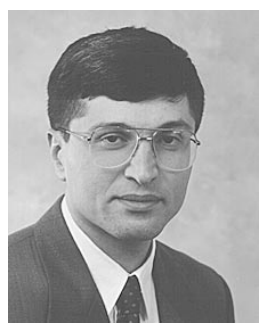

Farrokh Rashid-Farrokhi (S'88-M'97) received the B.S. and M.S. degree (highest honors) in electrical engineering from Sharif University of Technology, Tehran, Iran, in 1988 and 1992, respectively. In 1997, he received the Ph.D. degree in electrical engineering from the University of Maryland at College Park.

He joined the Wireless Communications Research Department, Bell Laboratories, Holmdel, NJ, as a Member of Technical Staff in 1998. His research interests include array and statistical signal processing, wireless communications, and networking.

Dr. Rashid-Farrokhi received the 1996-1997 George Harhalakis Outstanding Systems Engineering Graduate Student Award in recognition of outstanding contributions in cross-disciplinary research from the University of Maryland at College Park.

K. J. Ray Liu (S'86-M'90-SM'93), for a photograph and biography, see this issue, p. 1338 . 


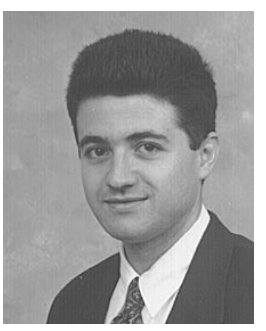

Leandros Tassiulas (S'89-M'91) was born in 1965, in Katerini, Greece. He obtained the Diploma in electrical engineering from the Aristotelian University of Thessalonikik, Thessaloniki, Greece in 1987 and the M.S. and Ph.D. degrees in electrical engineering from the University of Maryland, College Park, in 1989 and 1991, respectively.

From 1991 to 1995 he was an Assistant Professor in the Department of Electrical Engineering, Polytechnic University, Brooklyn, NY. In 1995 he joined the Department of Electrical Engineering, University of Maryland, College Park, where he is now an Associate Professor. He holds a joint appointment with the Institute for Systems Research and is a member of the Center for Satellite and Hybrid Communication Networks, established by NASA. His research interests are in the field of computer and communication networks with an emphasis on wireless communications (terrestrial and satellite systems) and high-speed network architectures and management, in control and optimization of stochastic systems, and in parallel and distributed processing.

Dr. Tassiulas received a National Science Foundation (NSF) Research Initiation Award in 1992, an NSF Faculty Early Career Development Award in 1995, and an Office of Naval Research Young Investigator Award in 1997. He coauthored a paper that received the INFOCOM '94 Best Paper Award. 